\title{
A Theorem on General Regular Transformations of Series
}

\author{
By Arwer Evans
}

(Received 11th December, 1950. Revised January, 1954.)

\section{Introduction.}

Many papers have been written on transformations of sequences which can be written as

$$
t_{m}=f_{m}\left(s_{1}, s_{2}, \ldots, s_{r}, \ldots\right),
$$

where $f_{m}\left(s_{1}, \ldots\right)$ is a function of a finite or infinite number of variables for fixed $m$. If the number of variables is finite it becomes large with $m$.

Almost all these papers are on linear transformations of the $s_{r}$, e.g. Cesàro and Abel means, but there are obvious transformations of nonlinear form which are regular, and it is a theorem on these which is considered here.

It is noteworthy that while the condition for regularity of a linear transformation can be expressed in terms independent of the sequence $s_{r}$, this is not true for non-linear transformations. Such a transformation may be regular only if $s$ and the $s_{r}$ lie in a restricted range (we assume $s_{r}$ real), as is shown in the examples below. To make this point clear we shall say that a transformation is regular in an interval $(a, b)$ (which to save complication shall be open) if it is regular for any sequence $\left(s_{r}\right)$ with limit $s$ when

$$
a<s_{r}<b, \quad a<s<b .
$$

\section{Examples of non-linear transformations.}

$[a]$ The geometric mean $t_{m}=\left(s_{1} s_{2} \ldots s_{m}\right)^{1 / m}$. Here $s_{r}>0$ and $s \geqslant 0$ for the transformation to be regular.

[b] $\quad t_{m}=\left(\frac{1}{m} \sum_{r=1}^{m} s_{r}^{k}\right)^{1 / k}$, where $k$ is real, $k \neq 0$ and $s_{r}>0$. 
An interesting particular case is when $k=-1$, as then we only need $s_{r} \neq 0$ for regularity.

[c] $t_{m}=\frac{1}{m}\left(\sum_{i, j, \ldots, l=1}^{m} s_{i} s_{j} \ldots s_{l}\right)^{1 / k}$, where $k$ is the number of suffixes and $s_{r} \geqslant 0$ for regularity.

We note that in all these transformations, if we put $s_{r}=S$, then $t_{m} \rightarrow S$, and also that a finite number of alterations do not affect $t_{m}$. It is on this idea that the theorem is based.

\section{Theorem and proof.}

If $t_{m}=f_{m}\left(s_{1}, s_{2}, \ldots, s_{m}\right)$, a real function of $m$ variables, then a sufficient condition for the transformation from the sequence $\left(s_{n}\right)$ to the sequence $\left(t_{m}\right)$ to be regular in an interval $(a, b)$ is that

(i) $f_{m}\left(s_{1}, s_{2}, \ldots, s_{k}, x, x, \ldots, x\right) \rightarrow x$ as $m \rightarrow \infty$, for all $x$ in $(a, b)$, where $k$ is a positive integer, and the $s_{n}$ are arbitrary numbers in $(a, b)$.

(ii) $f_{m}\left(s_{1}, s_{2}, \ldots, s_{m}\right)$ is an increasing function of each $s_{r}$ in the interval $(a, b)$ excepting a finite set of the $s_{r}$ independent of $m$.

Proof. Since $f_{m}\left(s_{1}, s_{2}, \ldots, s_{m}\right)$ is not monotonic for only a finite number of $s_{r}$ in $(a, b)$, there exists an $M$ such that when $m \geqslant M, f_{m}()$ is monotonic in the $s_{r}$ for $r \geqslant M$.

Suppose that $s_{r} \rightarrow s$; then for given $\epsilon>0$ there exists an $N=N(\epsilon)$ such that $\left|s_{r}-s\right|<\epsilon$ for $r \geqslant N$.

Now let $m>X=\max (M, N)$. Then since $f_{m}()$ is monotonic in the $s_{r}, r \geqslant M$, we have

$$
\begin{aligned}
f_{m}\left(s_{1}, s_{2}, \ldots, s_{X}, s-\epsilon, s-\epsilon, \ldots, s-\epsilon\right) & \leqslant f_{m}\left(s_{1}, s_{2}, \ldots, s_{X}, s_{X+1} \ldots, s_{m}\right) \\
& \leqslant f_{m}\left(s_{1}, s_{2}, \ldots, s_{X}, s+\epsilon, s+\epsilon, \ldots, s+\epsilon\right) .
\end{aligned}
$$

Therefore if $m>X$

$$
\begin{aligned}
&\left|f_{m}\left(s_{1}, \ldots, s_{m}\right)-s\right| \leqslant\left|f_{m}\left(s_{1}, s_{2}, \ldots, s_{X}, s-\epsilon, s-\epsilon, \ldots, s-\epsilon\right)-s\right| \\
&+\left|f_{m}\left(s_{1}, \ldots, s_{X}, s+\epsilon, s+\epsilon, \ldots, s+\epsilon\right)-s\right| \\
& \leqslant 2 \epsilon+\left|f_{m}\left(s_{1}, \ldots, s_{X}, s-\epsilon, \ldots\right)-(s-\epsilon)\right| \\
&+\left|f_{m}\left(s_{1}, s_{2}, \ldots, s_{X}, s+\epsilon, \ldots\right)-(s+\epsilon)\right| .
\end{aligned}
$$


But $X$ is fixed for given $\epsilon$ and $f_{m}\left(s_{1}, \ldots, s_{X}, s-\epsilon, s-\epsilon, \ldots, s-\epsilon\right) \rightarrow s-\epsilon$ as $m \rightarrow \infty$. Therefore there exists an $N_{1}$ such that if $m \geqslant N_{1}$

$$
\left|f_{m}\left(s_{1}, \ldots, s_{X}, s-\epsilon, \ldots, s-\epsilon\right)-(s-\epsilon)\right|<\epsilon .
$$

Similarly for the other term there exists an $N_{2}$. So if $m \geqslant \max \left(N_{1}, N_{2}\right)$

$$
\left|f_{m}\left(s_{1}, s_{2}, \ldots, s_{m}\right)-s\right|<4 \epsilon \text {. }
$$

But $\epsilon$ is arbitrary, and therefore $f_{m}\left(s_{1}, s_{2}, \ldots, s_{m}\right) \rightarrow s$ as $m \rightarrow \infty$.

\section{Notes.}

This theorem can be extended to the case where $f_{m}()$ is a function of an infinite number of variables: the statement and proof require no alteration. The proof requires only minor modifications when $(a, b)$ is the interval $(a, \infty)$ and $\lim s_{n}=\infty$. Similarly one may consider closed or semi-closed intervals of regularity with the appropriate minor modifications in the proof.

As the theorem in $§ 3$ is written it is obvious that condition (i) is necessary, but condition (ii) is not necessary although some such condition is needed. This follows from the known theory of linear transformations, see e.g. Hardy [2], p. 43, Theorem 2, or more clearly Widder [3], p. 115, Theorem 7 . 2. Our condition (i) is equivalent to (B) and (C) combined in [3], or to (3.2.4) and (3.2.5) in [2]. But for condition (A) or (3.2.3) in [2] and [3] we have no equivalent, condition (ii) being equivalent to the condition that $c_{m n} \geqslant 0$ for all sufficiently large $n$ independent of $m$, in the notation of [2] and [3].

Now as the Toeplitz conditions are both necessary and sufficient it is evident that without (ii) the theorem will cease to be true, and linear or non-linear examples are easily constructed to show this. But from the interpretation of the theorem in terms of linear transformations it is obvious that the condition as it stands is too strict.

A simple non-linear regular transformation which does not satisfy the theorem is provided by taking

$$
f_{m}\left(s_{1}, \ldots, s_{m}\right)=\left(\frac{s_{1} s_{2}}{s_{3}} \cdot \frac{s_{4} s_{5}}{s_{6}} \ldots \frac{s_{3 r-2} \cdot s_{3 r-1} \ldots s_{m}}{s_{3 r}}\right)^{3 / m},
$$

where $s_{r}>0$ and $\lim s_{r}>0$.

It can also be seen from the method of proof of the theorem that, for any transformation which satisfies the condition of the theorem,

$$
\underline{\lim } s_{n} \leqslant \underline{\lim } t_{m} \leqslant \varlimsup
$$


provided that $s, \varlimsup s_{n}, \underline{\lim } s_{n}$ are within the interval of regularity of the transformation. In fact the transformations which satisfy the conditions of the theorem can be regarded as generalisations of positive transformations mentioned in Hardy [2], p. 52.

A somewhat similar theorem has been proved by Sen-Ming Leng [1] although the conditions are different. The theorem is stated as follows:

Let $a_{n}$ be a sequence of real numbers convergent to $A: a_{n} \rightarrow A$. Let $f_{n}\left(\alpha_{1}, \alpha_{2}, \ldots\right)$ be a sequence of real-valued functions which is defined for any such sequence $\left(\alpha_{1}, \alpha_{2}, \alpha_{3}, \ldots\right)$ that, for some positive integer $h, \alpha_{v}=a_{\nu}$ or $A$ when $\nu \leqslant h$ and $\alpha_{v}=a_{\nu}$ when $\nu>h$. Suppose that each individual $a_{k}$ is ultimately immaterial to $f_{n}\left(a_{1}, a_{2}, \ldots\right)$ and $f_{n}\left(a_{1}, a_{2}, a_{3}, \ldots\right)$ is ultimately a mean, namely that

$$
\text { (1) } \begin{aligned}
\lim _{n \rightarrow \infty}\left[f_{n}\left(\alpha_{1}, \alpha_{2}, \ldots, \alpha_{k-1}, \alpha_{k}, \alpha_{k+1}, \alpha_{k+2}, \ldots\right)\right. \\
\left.-f_{n}\left(a_{1}, \ldots, a_{k-1}, A, \alpha_{k+1}, \alpha_{k+2}, \ldots\right)\right]=0
\end{aligned}
$$

and

(2) $\min \left(\alpha_{1}, \alpha_{2}, \ldots, \alpha_{k}, \alpha_{k+1}, \alpha_{k+2}, \ldots\right)+\eta_{n}$

$$
\begin{aligned}
& \leqslant f_{n}\left(\alpha_{1}, \ldots, \alpha_{k}, \alpha_{k+1}, \alpha_{k+2}, \ldots\right) \\
& \leqslant \max \left(\alpha_{1}, \ldots, \alpha_{k}, \alpha_{k+1}, \alpha_{k+2}, \ldots\right)+\zeta_{n}
\end{aligned}
$$

for every $\left(\alpha_{1}, \alpha_{2}, \alpha_{3}, \ldots\right)$ and for every positive integer $k$, where $\eta_{n} \rightarrow 0, \zeta_{n} \rightarrow 0$. Then

$$
f_{n}\left(a_{1}, a_{2}, a_{3}, \ldots\right) \rightarrow A \text {. }
$$

It can be seen that condition (1) corresponds to (B) in Widder [3], p. 115, but condition (2) here is completely different from any of the ones usually considered.

\section{REFERENCES.}

[1] Sen-Ming Leng, "A note on Cauchy's limit theorem ", American Math. Monthly, 57 (1950), 28-31.

[2] G. H. Hardy, Divergent Series (Oxford, 1949).

[3] D. V. Widder, The Laplace Transform (Princeton, 1946).

$$
\begin{aligned}
& \text { University of Western Ontario, } \\
& \text { London, Canada. }
\end{aligned}
$$

